\title{
Role of serum bilirubin as a marker of acute gangrenous appendicitis
}

\author{
Ghimire , $^{1^{*}}$ Thapa $P^{2}$ Yogi $N,{ }^{3}$ Ghimire $P^{4}$ \\ ${ }^{1}$ Department of Surgery, Manipal College of Medical Sciences, Pokhara, Nepal, ${ }^{2}$ Department of Psychiatry, Manipal College \\ of Medical Sciences, Pokhara, Nepal, ${ }^{3}$ National Institute of Neurological and Allied Sciences, Bansbari, Kathmandu, Nepal, \\ ${ }^{4}$ Medical Officer, Emergency Department, Manipal Teaching Hospital, Pokhara, Nepal
}

\section{*Corresponding Author:}

Dr. Pradeep Ghimire MS, FRCS Ed, Department of Surgery,

Manipal Teaching Hospital, Pokhara

Email: pradeepg757@hotmail.com

\section{Citation}

Ghimire $P$, Thapa $P$, Yogi $N$, Ghimire $P$. Role of serum bilirubin as a marker of acute gangrenous appendicitis. Nepal Journal of Medical sciences 2012;1(2):89-92.

\section{ABSTRACT}

Background: This study has compared the difference in serum bilirubin between patients with gangrenous and non-gangrenous appendicitis.

Methods: A prospective analytical study of, 141 patients who underwent appendectomy, from March 2010 to March 2011 in Manipal Teaching Hospital, Pokhara, Nepal was carried out. Clinico-demographic data, Alvarado's score, total serum bilirubin, total leucocyte count and histopathological report of all the cases were collected in a prestructured proforma. Comparison between gangrenous and non-gangrenous appendicitis groups was carried out using independent sample $t$ test, Chisquare test, and direct logistic regression. The data was analyzed using SPSS 11.6 software.

Result: Around 43\% (61 out of 141) patients had gangrenous appendicitis and statistically significant difference was found between the two groups in total Alvarado's score, total count and total bilirubin level. The effect size as indicated by eta square statistics was large for total bilirubin (eta squared $=0.39$ ) as compared to total Alvarado's score (0.09) and total leucocyte count (0.05). Direct logistic regression model showed serum total bilirubin as the only independent variable to make a unique statistically significant contribution in predicting gangrenous appendicitis.

Conclusion: Pre-operative assessment of serum total bilirubin can serve as an important maker of acute gangrenous appendicitis.

Keywords: Serum total bilirubin; acute gangrenous appendicitis; Alvarado's score

\section{Background:}

$\mathrm{A}_{\text {ppendectomy is one of the most commonly performed }}$ abdominal surgeries in an emergency setting. Delay in diagnosis and surgery for this condition may lead to various complications like perforation, abdominal abscess, urinary retention, small bowel obstruction and peritonitis causing an increase in morbidity and even mortality of the patients. On the other hand, too aggressive surgical approach may lead to an increased rate of negative appendectomies. The incidence of perforated appendicitis in adults has been reported from $13-37 \%$ or higher. ${ }^{1,2}$

Appendicitis has been shown to have a strong association with hyperbilirubenimia. It has been hypothesized that following appendicitis inflammatory agents like TNFalpha, IL-6 and cytokines usually migrate to the liver via the superior mesenteric vein producing inflammation, abscess 
or dysfunction of the liver function. These inflammatory agents may also alter the hepatic blood flow and normal physiological flow of the bile causing hyperbilirubinemia. ${ }^{3-8}$ Few other studies have compared various inflammatory agents with total bilirubin as a predictive marker of perforation of appendix.

Though various imaging modalities like computed tomography (CT) scan, magnetic resonance imaging (MRI) and ultrasonography may help in early diagnosis of perforated appendix, they may not be readily available in many health centers of the third world and developing countries. ${ }^{9-14}$ In such condition clinical and laboratory investigations may be the only, cheaper and readily available options for diagnosis. In this study we have tried to compare the difference in the level of serum bilirubin between patients with gangrenous and non-gangrenous appendicitis.

\section{Methods:}

A prospective analytical study was carried out comprising of a non-randomized cohort which was followed up from admission to the hospital till discharge in Manipal Teaching Hospital, Pokhara, Nepal. The sample comprised of 141 patients operated for appendicitis and confirmed by histopathological report in between March 2010 to March 2011.

Patients undergoing interval appendectomies, appendectomies for other indications and patient with negative appendectomies confirmed on histological report were excluded from the study. Other exclusion criteria were patient with risk factors for hepatic disease such as alcoholism, a history of viral hepatitis, Gilbert's disease, Dubin-Johnson syndrome, benign recurrent intra-hepatic cholestasis, and other documented biliary, haemolytic or liver diseases associated with hyperbilirubinaemia.

At admission demographic and clinical data, and the time period of symptoms' evolution, Alvarado's score, total leucocyte count and total serum bilirubin was recorded. All patients were followed-up during the postoperative period and any complication was recorded. After discharge from the hospital the histopathological report was recorded. The pathologist processing the specimen was not made aware about the clinical and operative details. The patients were divided in two groups; gangrenous and non-gangrenous according to the histological findings of the specimen.

Continuous variables were compared between the two groups using independent sample t test and the categorical variables were compared using chi square test. Direct logistic regression was performed to assess the impact of total Alvarado's score, total leucocyte count and serum direct bilirubin on the likelihood of having gangrenous appendicitis as compared to non-gangrenous appendicitis. All the data were analyzed using SPSS 11.6 software.

\section{Results:}

Out of a total of 141 patients included in the study 80 were classified as having non-gangrenous appendicitis and 61 were classified as having gangrenous appendicitis based on biopsy reports. Ages, total Alvarado's score, total count, duration of pain, total bilirubin level between the groups were compared using independent sample t test. Statistically significant difference was found between the groups in total Alvarado's score, total count and total bilirubin level. Difference in age and duration of pain was not statistically significant (table 1).

Table 1: Comparison of different variables using independent sample $t$ test

\begin{tabular}{lccccccc}
\hline Variables & \multicolumn{2}{c}{$\begin{array}{c}\text { Mean (SD) } \\
\text { Gangrenous } \\
\text { Nongangrenous }\end{array}$} & $\begin{array}{c}\mathrm{T} \\
\text { value }\end{array}$ & df & P value & $95 \%$ CI \\
\hline Age & 32.52 & 29.69 & 0.95 & 108.85 & 0.342 & -3.09, \\
& $(19.58)$ & $(14.95)$ & & & & 8.84 \\
Total & 8.25 & 7.19 & 3.79 & 136.14 & $<0.001$ & 0.50, \\
Alvarado's & $(1.54)$ & $(1.75)$ & & & & 1.61 \\
Total & 13398 & 11784.73 & 2.71 & 139 & 0.008 & 436.39 \\
Count & $(3513.43)$ & $(3492.89)$ & & & & 2790.15 \\
Duration of & 38.92 & 37.89 & 0.23 & 139 & 0.817 & -7.57, \\
Pain & $(25.21)$ & $(26.83)$ & & & & 9.81 \\
Total & 1.76 & 1.07 & 9.60 & 80.99 & $<0.001$ & 0.54, \\
Bilirubin & $(0.52)$ & $(0.24)$ & & & & 0.83 \\
\hline
\end{tabular}

The effect size as indicated by eta square statistics was small for total Alvarado's score and total count (eta squared= 0.09 and 0.05 respectively) and large for total bilirubin (eta squared $=0.39$ ). The sex difference between the groups was compared using chi square test and the difference between the group was statistically not significant $\mathrm{p}=0.284$ (Table 2 ).

Table 2: 2X2 Table for sex distribution among two groups

\begin{tabular}{lccccc}
\hline Sex & Gangrenous & $\begin{array}{c}\text { Nongan- } \\
\text { grenous }\end{array}$ & $\begin{array}{c}\text { Chi } \\
\text { Value }\end{array}$ & df & P value \\
\hline Male & 33 & 36 & & & \\
Female & 28 & 44 & 1.14 & 1 & 0.284 \\
Total & 61 & 80 & & & \\
\hline
\end{tabular}

Direct logistic regression was performed to assess the impact of total Alvarado's score, total count and direct bilirubin on the likelihood of having gangrenous appendicitis as compared to non-gangrenous appendicitis.

The full model containing all the predictors was statistically 
significant; chi square $(3, \mathrm{~N}=141)=80.1, \mathrm{p}<0.001$ indicating that the model was able to distinguish between gangrenous and non-gangrenous appendicitis. The model as a whole explained between $43.3 \%$ (Cox \& Snell R square) and 58.1\% (nagelkelrke R squared) of variance. As seen from table 3; only one independent variable made a unique statistically significant contribution to the model i.e. total bilirubin.

Table 3: showing results of logsistic regression analysis

\begin{tabular}{lccccc}
\hline \multicolumn{1}{c}{ Variables } & B & S.E & Wald & Df & P value \\
\hline Total Alvarado's & 0.02 & 0.15 & 0.01 & 1 & 0.895 \\
Total Count & 0.00 & 0.00 & 0.09 & 1 & 0.756 \\
Total Bilirubin & 4.51 & 0.76 & 34.63 & 1 & $<0.001$ \\
Constant & -6.77 & 1.33 & 25.90 & 1 & $<0.001$ \\
\hline
\end{tabular}

\section{Discussion:}

Appendicitis is the most common surgery performed for acute abdominal pain. Delay in surgery in these cases due to any reasons; delayed presentation or mistaken judgment, leads to dreaded complications like gangrenous changes and perforation. The rate of morbidity and mortality for patients operated for perforated or gangrenous appendicitis is much higher than those operated for non-gangrenous appendicitis. Diagnosis of appendicitis and even perforated appendicitis is entirely based on clinical assessment and judgment of the treating surgeon. Various radiological investigations like USG and multidetector CT scans can detect appendicitis with high precision but they are very unspecific; the diagnosis of perforation can be made only when there is abscess or extra luminal gas present in the peritoneal cavity.

Recently few studies have investigated the role of serum bilirubin in diagnosis of perforated appendicitis. One study has found hyperbilirubinemia to be an important predictive marker for perforated and necrotizing appendicitis. In this series of 157 patients, the odds of gangrenous and perforated appendicitis was found to be 3 times higher for patients having hyperbilirubinemia than those with normal bilirubin level. ${ }^{15}$ Similarly, another study investigated 538 patients to determine the value of serum bilirubin in diagnosing acute suppurative or necrotizing appendicitis and found that the specificity of serum bilirubin was higher $(86 \%)$ than other markers like WBC (55\%) and C-reactive protein (CRP) (35\%) in diagnosing gangrenous and perforated appendicitis. ${ }^{16}$ A retrospective analysis including 351 cases of acute appendicitis investigating preoperative markers for perforated appendicitis found that symptom duration $(p=0.002)$, total bilirubin $(p=0.000)$ and elevated leucocyte counts $(p=0.011)$ were significant independent variables for identifying acute perforated appendicitis against the suppurative ones. ${ }^{17}$

A retrospective cohort study performed in two centers involving 155 cases of perforated appendicitis and 570 cases of non-perforated appendicitis compared hyperbilirubinemia, CRP, leucocyte count and age as a marker of perforated appendicitis. They came to the conclusion that hyperbilirubinemia is a statistically significant marker of perforated appendicitis but they also found CRP to be a superior marker of perforation than hyperbilirubinemia. ${ }^{18}$

In our study there was statistically significant difference between the gangrenous and non-gangrenous groups in total Alvarado's score, total count and total bilirubin level. Difference in age and duration of pain was not statistically significant. The effect size as indicated by eta square statistics was small for total Alvarado's score and total count (eta square $=0.09$ and 0.05 respectively) and large for total bilirubin (eta squared $=0.39$ ). Direct logistic regression indicated that only one independent variable made a unique statistically significant contribution to the model i.e. total bilirubin. Our results are more or less consistent to the results obtained by various other studies mentioned above but there were few limitations; we did not include other indicators like CRP in the study.

\section{Conclusion:}

Preoperative assessment of serum bilirubin should be routinely performed in cases of acute appendicitis. It can not only help in diagnosis of acute appendicitis but can also serve as an important maker of acute gangrenous appendicitis.

\section{Conflict of interest: none}

\section{References:}

1. Temple CL, Huchcroft SA, Temple WJ. The natural history of appendicitis in adults - A prospective study. Ann Surg 1995;221:278-81.

2. Korner H, Sondenaa K, Soreide JA, et al. Incidence of acute non-perforated and perforated appendicitis: Age-specific and sex-specific analysis. World J Surg 1997;21:313-7.

3. Beg RB, Garlungton AW. Translocation of certain endogenous bacteria from the GI tract to mesenteric lymph node and other organ in Gonobiotic mouse model. Infect Immunol 1979;23:403-11.

4. Juric I, Primorac D, Zagar Z, et al. Frequency of portal and systemic bacteremia in acute appendicitis. Pediatr Int 2001;43:152-6.

5. Koito Scathen WE, Desprez JD, Holden WD. A bac- 
teriologic study in portal blood in man. Arch Surg 1995;71:404-9.

6. Wang P, Ayala A, Ba ZF, et al. Tumor necrosis factor -alpha produces hepatocellular dysfunction despite of normal cardiac output and hepatic microcirculation. Am J Physiol Gastrointet Liver Physiol 1993:265:126-32.

7. Wang P, Ba ZF, Chaudhary IH. Hepatic extraction of indo-cyanine green is depressed in early sepsis despite incre ased hepatic blood flow and cardiac output. Arch Surg 1991;2:126.

8. Whiting JF, Green RM, Rosen AB, et al. TNF-alpha decreases hepatocyte bile salt uptake and mediated endotoxin-induced cholestasis. Hepatol 1995; 22:1273.

9. Amalesh T, Shankar M, Shankar R: CRP in acute appendicitis - is it a necessary investigation? Int J Surg 2004;2:88-9.

10. Khan MN, Davie E, Irshad K: The role of white cell count and C-reactive protein in the diagnosis of acute appendicitis. J Ayub Med Coll Abbottabad 2004;16:179.

11. deDombal FT, Leaper DJ, Staniland JR, et al: Computer-aided diagnosis of acute abdominal pain. BMJ 1972;2:9-13.
12. Balthazar EJ, Megibow AJ, Siegel SE, et al: Appendicitis: prospective evaluation with high resolution $\mathrm{CT}$. Radiology 1991;180:21-4.

13. Rao PM, Boland GW: Imaging of acute right lower abdominal quadrant pain. Clin Radiol 1998;53:639-49.

14. Bixby SD, Lucey BC, Soto JA, et al: Perforated versus nonperforated acute appendicitis: accuracy of multidetector CT detection. Radiology 2006;241:780-6.

15. Estrada JJ, Petrosyan M, Barnhart J, et al. Hyperbilirubinemia in appendicitis: A new predictor of perforation. J Gastrointest Surg 2007;11:714-8.

16. Sand M, Bechara FG, Holland-Letz T, et al: Diagnostic value of hyperbilirubinemia as a predictive factor for appendiceal perforation in acute appendicitis. Am J Surg 2009;198:193-8.

17. Atahan K, Ureyen O, Aslan E, et al. Preoperative diagnostic role of hyperbilirubinaemia as a marker of appendix perforation. The Journal of International Medical Research. 2011;39:609-18.

18. Käser SA, Fankhauser G, Willi N, et al. Creactive protein is superior to bilirubin for anticipation of perforation in acute appendicitis. Scand J Gastroenterol 2010;45:885-92. 